Slavica

bruxellensia

\section{Slavica bruxellensia}

Revue polyphonique de littérature, culture et histoire

slaves

$8 \mid 2012$

Migration(s) et Exil(s)

\title{
La double identité culturelle de Paul Cazin
}

(1881-1963)

\section{Danuta Knysz-Tomaszewska}

\section{OpenEdition}

\section{Journals}

Édition électronique

URL : http://journals.openedition.org/slavica/1065

DOI : 10.4000/slavica.1065

ISSN : 2034-6395

\section{Éditeur}

Université libre de Bruxelles - ULB

\section{Référence électronique}

Danuta Knysz-Tomaszewska, «La double identité culturelle de Paul Cazin (1881-1963) », Slavica bruxellensia [En ligne], 8 | 2012, mis en ligne le 15 juin 2012, consulté le 19 avril 2019. URL : http:// journals.openedition.org/slavica/1065; DOI : 10.4000/slavica.1065

Ce document a été généré automatiquement le 19 avril 2019

\section{(c) $(1) \odot$}

Les contenus de Slavica bruxellensia sont mis à disposition selon les termes de la Licence Creative Commons Attribution - Pas d'Utilisation Commerciale - Pas de Modification 3.0 France. 


\section{La double identité culturelle de Paul Cazin (1881-1963)}

Danuta Knysz-Tomaszewska

Introduction 
1 Cette étude est consacrée à l'analyse de la situation équivoque dans laquelle s'est trouvé Paul Cazin, écrivain français devenu l'un des meilleurs spécialistes de la littérature polonaise de son temps et a contribué à mieux la faire connaître en France. Ses activités de traducteur et de conférencier semblent éclipser sa propre création littéraire aux yeux de ses compatriotes. La perspective que j'ai adoptée s'intéresse en priorité au décalage

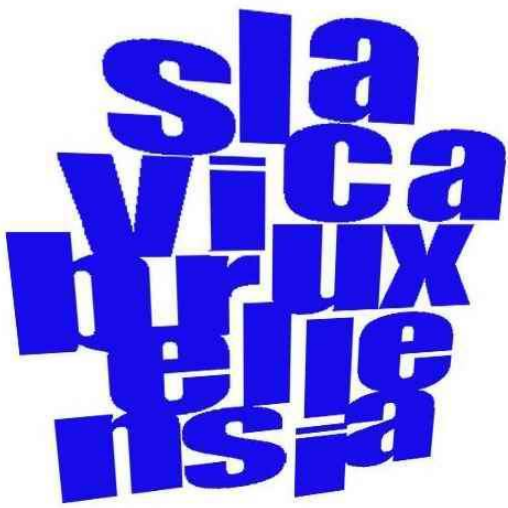
de sa renommée en Pologne et en France et aux conséquences de ce hiatus. La base et le point de départ essentiel de mon étude est constitué par les archives privées de Cazin : ses lettres, son Journal d'Autun (1925-1949), dont je signale les lacunes, ainsi que son Journal d'Aix-en-Provence (1953-1963). Je me suis également servie des coupures de presse conservées par Cazin lui-même et par sa famille après sa mort ainsi que de la documentation qui m'a été gracieusement transmise par «L'Association des Vieux Parodiens » de Paray-le-Monial et par feu Marcel Barbotte, président de l'Association des Amis de Paul Cazin, créée à Autun après la mort de l'écrivain ${ }^{1}$. J'ai également profité de la bibliographie des textes de Cazin publiés dans la presse française et polonaise établie par Kazimierz Chruściński².

2 Marginalisé, plutôt oublié par les critiques français, Cazin est apprécié à sa juste valeur et même vénéré en Pologne. Il s'inscrit dans la lignée de ces artistes dont la double identité culturelle et la double passion créative constituent à la fois la richesse et le danger. Pendant toute sa vie, il s'est senti déchiré entre ses traductions d'œuvres polonaises et sa carrière d'écrivain français, dont il rêvait, disons-le, en vain, malgré le succès de ses deux premiers livres L'Humaniste à la guerre (1920) et Décadi (1921).

3 Les contradictions embrassent différents domaines de sa vie. Profondément provincial, amoureux du pays de son enfance et de la prestigieuse ville de Paray-le-Monial, il doit se partager entre Paris et Autun, où il a passé presque trente ans. Fidèle à la Bourgogne, très sensible à la beauté du Morvan, il se voit obligé d'aller vivre en Provence vers la fin de l'année 1949 pour assurer son existence, celle de sa deuxième épouse et de ses deux fillettes. Il se sentira toujours déraciné et exilé à Aix-en-Provence.

Il n'a pas réussi à se frayer de chemin parmi les écrivains français des années 1930. Malgré son doctorat ès lettres obtenu à Lviv en 1932, la France ne pouvait lui offrir de poste de polonisant dans l'une de ses universités. Il a fallu attendre la décision du gouvernement de la Pologne Populaire qui, en 1950, a créé pour lui un poste de lecteur de polonais à l'Université d'Aix-en-Provence. Les échecs auprès des éditeurs et de la critique française ont condamné Cazin à une sorte d'exil psychique. Tout en restant dans le Sud de la France, il vivait au travers de la culture et de la littérature polonaise. Tous ses succès étaient liés à la Pologne.

5 Quelle ironie du sort et quel concours de circonstances historiques! La protection d'un pays communiste ainsi que les liens qui l'unissaient avec les écrivains polonais l'ont compromis aux yeux de l'émigration, groupée autour de l'intransigeante Bibliothèque Polonaise à Paris. La Bibliothèque a rompu ses relations avec Cazin, qui y avait pourtant 
assuré des cours de littérature et de civilisation polonaises dans les années 1935-1939. Il me parait important de souligner que les conséquences de ces perturbations politiques se sont avérées graves. Après 1949, le champ français d'activités intellectuelles et littéraires accessible à Cazin s'est considérablement rétréci.

« Déporté » en Provence, oublié par les éditeurs, rejeté par la droite du Paris polonais et soumis à une sorte d'ostracisme, Cazin est devenu un «étranger ", un « émigré » dans son propre pays. Tel est le prix qu'il a dû payer pour sa double identité culturelle et la fidélité à ses amis de l'autre coté du rideau de fer. L'humaniste, l'érudit, plongé dans le monde des classiques et dans celui d'une littérature inconnue des Français, se trouve esseulé dans la Provence ensoleillé et abandonné par ses anciens collaborateurs du Centre d'Études polonaises de l'Ile Saint-Louis. Il n'a jamais voulu faire de politique mais la politique s'est emparée de son sort.

7 En revanche, en Pologne, sa patrie adoptée, il jouit d'un prestige aussi bien pendant l'entre-deux-guerres qu'après 1945. Il est possible de constater que Cazin trouve un grand réconfort moral dans sa gloire d'ambassadeur de la culture polonaise, qui lui permet de poursuivre ses activités intellectuelles malgré les réticences des maisons d'édition. Le poste universitaire subventionné par le gouvernement polonais lui permet également de subvenir aux besoins de sa famille agrandie. C'est pourquoi il est possible de voir en lui un exilé, bien qu'il soit resté dans cette belle ville d'Aix, qui n'est jamais devenue la sienne. Le fait qu'il ait voulu recevoir son titre d'officier de la Légion d'Honneur à Paray-leMonial en 1959 n'est pas sans importance. Et il est très significatif que sa dernière volonté fut qu'on l'enterre dans cette ville, dont il a chanté la paisible beauté dans Décadi.

Le souvenir de Cazin, écrivain français ettraducteur de littérature polonaise, reste toujours vivant dans la mémoire des Polonais. Il était considéré comme le premier grand traducteur français, passionné par la langue et la culture polonaises. Pendant plus de cinquante ans, il a cherché à familiariser ses compatriotes avec des œuvres datant du XVII ${ }^{e} \mathrm{au} \mathrm{Xx}{ }^{\mathrm{e}}$ siècle. En polonisant compétent, il connaissait bien l'histoire de la Pologne, ses relations intellectuelles avec l'Europe Occidentale et la spécificité de sa littérature, qui, pendant 123 ans, devait véhiculer le sentiment d'identité nationale. Ses traductions de Józef Weyssenhoff, Władysław Reymont, Jan Chryzostom Pasek ou de Wacław Berent témoignent d'un art rare ainsi que de sa parfaite maitrise de la langue polonaise de différentes époques. Il manipulait aussi bien la langue baroque desPamiĘtniki (traduit par Les Mémoires en 1922 ${ }^{3}$ ) de Pasek que les exubérances stylistiques de Reymont dans $Z$ ziemi chetmskiej (traduit par L'Apostolat du knout en Pologne. Notes de voyage au Pays de Chelm en $1912^{4}$ ), ou encore la discipline intellectuelle du style de Tadeusz Breza dans Spiżowa brama (traduit par La Porte de Bronze en 19625) ou dans UrzĄd(traduit par La Démarche en19636). Il a accepté le défi des grands textes romantiques en traduisant Pan Tadeusz d'Adam Mickiewicz (19347), Stygmat (traduit par Le Stigmate en $1932^{8}$ ) de Cyprian Kamil Norwid, les œuvres de Zygmunt Krasiński ou de Juliusz Słowacki. Certains textes sont longtemps restés inédits comme Nie-Boska komedia (traduit par La Comédie non divine ) de Z. Krasiński, qui n'a été publiée qu'en l'an 2000, par les soins de Christophe Jeżewski. L'effort gigantesque de Cazin-traducteur nous réserve encore de nombreux textes à découvrir dans ses papiers ou dans la presse de l'époque ${ }^{10}$. Tout comme ses archives qui peuvent nous révéler beaucoup de secrets cachés dans ses lettres et sur les feuilles jaunies de son Journall ${ }^{11}$. 


\section{Cazin et les Polonais - des affinités d'âme et d'esprit}

9 Les critiques et les écrivains polonais n'ont pas manqué de constater qu'il existait une sorte de parenté spirituelle entre Cazin et les Polonais. Plusieurs facteurs peuvent expliquer cette affinité. Citons sa formation classique, son catholicisme fervent bien que peu docile, sa profonde conviction que la littérature est chargée d'une mission vis-à-vis de son peuple, son charmant provincialisme qui se révèle dans l'enracinement dans sa terre de Bourgogne. Il est proche des Polonais par son patriotisme qui l'a mené dans les tranchées de la Grande Guerre, évoquées dans L'Humaniste à la guerre. Ce patriotismel'a également dirigé vers les réseaux de la Résistance dans la zone occupée durant la Deuxième Guerre mondiale, pour en faire finalement le chroniqueur intrépide de la bataille d'Autun en août 1944. En témoin scrupuleux, il notait les événements au jour le jour, plus axé sur leur dimension historique que sur sa participation personnelle. Il n'est pas étonnant que La Bataille d'Autun, publiée en 1946, ait servi de base pour les futurs historiens et documentalistes qui ont écrit l'histoire de ces jours sanglants et héroïques de l'insurrection et de la libération de la ville par les Autinois ${ }^{12}$.

Les Polonais ont immédiatement deviné qu'il était capable de s'identifier avec leur culture et leur tempérament. Tadeusz Boy-Żeleński a déclaré en 1934 :

Bien que je n'aie vu Cazin que deux ou trois fois à peine, j'ai l'impression de le connaître depuis des temps immémoriaux, et très intimement. La raison en est peut-être une passion commune. Jadis, au début de notre connaissance par correspondance, Cazin m'écrivait: "Des affinités plus étroites que celles qui unissent les gens de la même nationalité et langue existent entre nous : nous avons chacun deux âmes pour se comprendre. $»^{14}$

Rappelons en plus qu'à partir de 1910, Cazin signait souvent ses lettres Paweł Kaziński, déclarant ainsi son appartenance aux deux sources culturelles.

11 Il a d'ailleurs évoqué à plusieurs reprises sa double nationalité spirituelle et notait scrupuleusement dans son Journal toutes les preuves d'estime et d'amitié dont il jouissait en Pologne.

\section{Retour aux sources de la grande aventure polonaise de Cazin}

12 Le pur hasard a voulu que le jeune licencié de la Faculté de Lettres de la Sorbonne, timide et sérieux, soit devenu un polonisant fervent. Son premier contact direct avec des Polonais, la famille Raczyński, date de 1904 et son premier voyage en Pologne a eu lieu en 1905 quand il est venu à Rogalin comme précepteur des jeunes fils du comte Edward Raczyński père. Cazin, modeste ressortissant de la petite ville bourguignonne de Paray-leMonial et moine manqué des franciscains, est entré dans la nouvelle vie intellectuelle par le palais de Rogalin, son milieu d'élite raffinée et ses mécènes d'art. Entouré de gens bienveillants qui savaient apprécier son intelligence et ses larges connaissances littéraires, Cazin a pu découvrir la langue polonaise dont la mélodie l'enchantait. La somptueuse bibliothèque du palais de Rogalin lui a offert un premier contact avec la littérature polonaise. Raczyński père est devenu un ami et un protecteur qui l'a aidé financièrement jusqu'à la Première Guerre mondiale, ce que confirme une lettre 
d'Edward Raczyński fils à Jerzy Borejsza de $1993^{15}$. Le fils a consacré à Cazin quelques tendres souvenirs dans ses mémoires de jeunesse à Rogalin ${ }^{16}$.

Les séjours de Cazin chez les Raczyński en 1905 et 1906 ainsi que ses voyages à Cracovie, Lviv et Laszki ont décidé de son avenir de traducteur et de polonisant. C'est en 1906 que Cazin a declaré dans une lettre à Adam Krasiński :

Je laisse dormir au fond de ma malle ma littérature, ma philosophie et tous les adjectifs à l'aide desquels je m'étais naïvement promis de faire une profonde impression sur mes contemporains (...). Je m'intéresse toujours néanmoins aux traductions et $\mathrm{j}$ 'espère parer avec autant de grâce que possible les enfants des autres. ${ }^{17}$

Fasciné par les trésors de la culture et de la littérature polonaise qu'il vient de découvrir, Cazin veut en faire bénéficier le public français. Toutefois cette décision résulte en partie du peu de confiance en soi et en ses capacités littéraires. Dans ce passage se dessine déjà la préfiguration du drame intérieur de Cazin dont ses amis polonais ne se sont jamais rendu compte. Néanmoins le Journal et certaines lettres destinées à ses correspondants français nous dévoilent que pendant toute sa vie Cazin était déchiré entre sa propre création et son travail de traducteur, considéré en France comme une activité secondaire. Dans une enquête de La Pologne Littéraire de 1933 Cazin avoue :

J'ai toujours considéré la traduction comme une besogne servile, mettez, si le mot vous semble trop dur, subalterne, utilitaire, dont le but est avant tout de fournir des documents d'étude ou d'interprétation. (...) C'est pour ne pas trop estropier les gentillesses que la littérature polonaise envoie par mon entremise à la française que je m'applique à faire œuvre d'art. ${ }^{18}$

Malgré le succès de la publication de Pan Tadeusz (nota bene traduit en prose), et de ses trois éditions consécutives (1934, 1936, 1937), Cazin n'a pas changé d'opinion en disant : « Être un traducteur cela veut dire consacrer ses ambitions personnelles et offrir tout son art au service d'un autre. $»^{19}$

Dans une certaine mesure, cette année 1906 a déterminé non seulement l'avenir de Cazin mais également la fortune de la littérature polonaise en France dans les trois premières décennies $\mathrm{du} \mathrm{xx}^{\mathrm{e}}$ siècle. Il est important de souligner que le cheminement des œuvres polonaises en France s'est avéré plutôt difficile. La carrière de traducteur de cette littérature "exotique "s'est révélée épineuse et pleine d'embûches, tant à cause des questions politiques dues au rapprochement de la France et de la Russie au début du xx siècle qu'en raison du peu d'intérêt porté par les lecteurs français à cette région qui n'existait pas sur la carte politique de l'Europe. La littérature polonaise ne promettait guère de succès pécuniaires à son adepte. En 1915, alors qu'il est déjà enrôlé dans l'armée, Cazin publie une ambitieuse étude intitulée "Le roman polonais au XIX siècle ». Il y constate avec une ironie grinçante que le bassin de la Vistule paraît pour un Français plus exotique que les antipodes (l'Extrême-Orient ou la Sibérie lointaine) décrits par Wacław Sieroszewski ${ }^{20}$. Il en parle en bonne connaissance de cause parce qu'il a déjà traduit Modlitwa Pańska (traduit par Oraison Dominicale en $1908^{21}$ ) de Gabriela Zapolska, Żywot $i$ myśli Zygmunta Podfilipskiego (traduit par Vie et opinions de Sigismond Podfilipski en 191022) de Weyssenhoff, $Z$ ziemi chetmskiej (traduit par L'Apostolat du knout en Pologne. Notes de voyage au Pays de Chelm en 1912 23 ) et Sprawiedliwie (traduit par Justice en 1912 ${ }^{24}$ ) de Reymont ainsi que Cień (traduit par L'Ombre), la première partie de Uroda życia (La Beauté de la vie) de Stefan Żeromski, publiée en 1912 dans La Vie, la revue de Marius et Ary Leblond ${ }^{25}$.

Dans la période qui précédait la Première Guerre mondiale, Cazin a rejoint l'Agence polonaise de presse à Paris puis le Comité franco-polonais (1909) et il s'est lié d'amitié 
avec Kazimierz Woźnicki qui, comme le décrit bien Franciszek Ziejka ${ }^{26}$, savait mobiliser beaucoup de Français du monde intellectuel à Paris autour de la Cause polonaise. Dans ce milieu, Cazin a rencontré le slavisant Ernest Denis, le critique Gabriel Sarrazin, le sculpteur Antoine Bourdelle ainsi qu'un grand nombre d'écrivains polonais tels que Reymont, Żeromski ou Wacław GĄsiorowski. Ziejka, spécialiste du Paris polonais, constate très justement que «Cazin est devenu pour la majorité des écrivains polonais un grand espoir de gagner le milieu des lecteurs français $» .^{27}$

C'était une chance pour Cazin, traducteur qui s'est alors mis à cette tâche avec ardeur; il pensait aussi au doctorat de littérature polonaise, visant la vague possibilité d'une chaire de littérature polonaise à la Sorbonne ou à L'École des Langues Vivantes à Paris. Il semble qu'avant 1914, l'avenir de Cazin traducteur et polonisant était décidé. Au début du xx siècle, Cazin a joué un rôle prépondérant dans la construction des liens culturels entre la Pologne et la France. Ses liens sont devenus le point de départ de vives relations littéraires et culturelles entre les deux pays pendant les années 1920 et 1930.

\section{Un choix difficile entre les carrières d'écrivain français et de traducteur}

17 Si l'histoire a interrompu et retardé la carrière universitaire de Cazin polonisant, elle a eu le mérite d'avoir éveillé en lui l'écrivain français. C'est en 1920 qu'il a publié L'Humaniste à la guerre, livre qui découlait de lettres à sa femme et de notes journalières prises au front en 1915. Cet ouvrage lui a valu le Prix « Marcel Guérin» de l'Académie Française et une haute opinion de Jacques Maritain qui constate : «J'aime L'Humaniste à la guerre parce que je vois briller en lui la perfection spirituelle de la mesure et du nombre. $»^{28} \mathrm{Et}$ il ajoute ensuite: "Mais je l'aime aussi parce qu'au souvenir des Muses viennent sans cesse se mêler en lui les échos du chant sacré, et parce qu'il nous apporte la promesse d'un humaniste chrétien renouvelé. $»^{29}$

18 Jean Norton Cru, un grand spécialiste des journaux et des mémoires de la Première Guerre mondiale, a lui aussi reconnu les valeurs humaines et littéraires de ce livre. Il a écrit dans sa monographie des souvenirs de combattants : «Le livre de Cazin est un régal pour les esprits délicats, épris aussi de vérité. Il est à la fois tragique, savamment spirituel et fort amusant. Cette antinomie qui est celle de la vie humaine constitue proprement l'humour et l'intelligence de Cazin. $»^{30}$

Ce premier grand succès auprès des critiques lui a rendu confiance en son potentiel créatif. Après douze ans d'apprentissage littéraire en tant que traducteur, Cazin débute comme un écrivain mûr, doté d'un style raffiné, très personnalisé, fidèle à sa biographie intellectuelle et à ses liens avec la Bourgogne. Jusqu'en 1928, il se concentre surtout sur ses propres œuvres qu'il tisse le plus souvent de ses souvenirs comme le roman Décadi ou la pieuse enfance (1921) qui évoque ses jeunes années à Paray-le-Monial. Ses écrits se nourrissent de ses lettres, de son Journal, de ses lectures classiques ainsi que des observations de la vie quotidienne en province où il s'installe définitivement dans la belle ville d'Autun à partir de 1924.

Les petits volumes de Cazin tels que L'Alouette de Pâques (1924), Les Lubies (1927) puis La Tapisserie des jours (1934) prennent la forme d'essais et de contes, esquissés d'une plume délicate et raffinée en forme de dialogue latent du narrateur avec lui-même et avec le lecteur. L'écrivain français l'emporte sur le traducteur. Cazin abandonne les traductions 
littéraires pour quelques années mais ses liens avec la littérature polonaise ne s'effacent pas puisqu'en 1922, il peut enfin voir l'édition des Mémoires de Jean-Chrysostome Pasek, Gentilhomme polonais (1656-1688), qu'il a traduits avant la guerre. Son introduction et ses notes témoignent de la bonne connaissance de la littérature et de l'histoire de la Pologne. L'Académie Française couronne son savoir et son talent d'un prix prestigieux.

Un grand voyage en Pologne en 1928 l'a réconcilié avec son rôle de traducteur et de polonisant. Il a été reçu avec toutes les preuves d'amitié et d'estime. Applaudi, attendu partout, recherché et invité dans le milieu de l'élite artistique et intellectuelle, il a pu renouveler ses contacts amicaux avec la famille Raczyński et avec Weyssenhoff. Il s'est lié d'amitié avec un éminent écrivain de Lviv, Parandowski. Il a rencontré des personnalités célèbres telles que le peintre Józef Mehoffer, le sculpteur Xavery Dunikowski et le dramaturge Hieronim Morstin, qui l'a invité dans son domaine de Pławowice en juin 1928 pour une rencontre avec des poètes polonais.

Ébloui par son succès, Cazin écrit dans une lettre à sa femme le 8 juin 1928 :

On m'a dit ce soir que jamais aucun Français n'avait été reçu ici avec une sympathie aussi profonde. On en avait reçu avec pompe, politesse ou amitié, jamais avec une pareille tendresse. Je suis littéralement noyé de cartes, de cadeaux, d'invitations. ${ }^{31}$

Durant son voyage, Cazin donne de nombreuses conférences, visite plusieurs villes, fait des rencontres excitantes, savoure sa gloire mais n'oublie jamais que ses propres livres se vendent plutôt mal, que les éditeurs français ne le recherchent pas et qu'il ne gagne pas bien sa vie. Le décalage entre la gloire dont il jouit en Pologne et sa position marginale dans la littérature contemporaine française font naître à la fois des réflexions douloureuses et de l'espoir pour le futur. Cazin croit que sa gloire polonaise pourrait conjurer le mauvais sort en France.

Ses admirateurs polonais, qui croient que son talent de traducteur le fera entrer dans la littérature européenne, ne se rendent compte ni de son déchirement pénible ni de sa pauvreté matérielle. Dans le message déjà cité du 8 juin 1928, Cazin écrit : «En somme, pour me faire connaître et mes livres, ce voyage, crois-le, aura été excellent. Amie chérie, je me sens tout encouragé. Je ferai quelque chose. \$ $^{32}$

25 La lecture de ses lettres de 1928 ainsi que de son Journal laisse deviner que la carrière de traducteur jouait pour lui le rôle d'un tremplin pour vaincre une certaine indifférence des milieux littéraires français. La gloire acquise en Pologne aurait pu lui servir à trouver une place parmi les écrivains de son pays.

26 La réponse à une enquête d'Henri Corbier en 1930 atteste de la déception et du découragement de Cazin qui constate avec mélancolie :

Aucun de mes ouvrages n'a connu le succès de vente. Aucun n'a été rémunéré je ne dis pas au tarif de maçons ou des frotteurs de parquet, mais au tarif de femme de ménage de nos petites villes de province. Du reste ils ne peuvent convenir au goût du grand public. (...) Je n'ai donc pas pu vivre de ma plume. Mais on peut vivre de la plume de tout le monde en écrivant des articles, des études, des traductions. ${ }^{33}$

Il serait difficile de ne pas déceler une certaine résignation dans cet aveu.

\section{Les années de plénitude et d'harmonie}

Il me semble que dans les années 1930, Cazin arrive à harmoniser au prix d'un effort immense le travail de traducteur, d'écrivain et de polonisant. Il a soutenu sa thèse de doctorat sur l'œuvre d'Ignacy Krasicki à l'Université de Lviv en 1932 et devait le refaire à 
Paris en 1939, mais la guerre a retardé cette soutenance à la fin de 1949 (Lyon). ${ }^{34}$ Ses rêves d'enseignant universitaire se sont réalisés au bout de trente-six ans puisqu'il a obtenu son poste de lecteur subventionné par l'Ambassade de Pologne Populaire en 1950 à l'Université d'Aix-en-Provence. L'histoire du $\mathrm{xx}^{\mathrm{e}}$ siècle est atrocement intervenue dans sa carrière universitaire, mais les bases de cette carrière ont été construites au début des années 1930.

Sa propre œuvre littéraire révèle plusieurs motifs polonais qui confirment cette nouvelle harmonie. Elle trouve l'expression la plus profonde dans l'infiltration du monde polonais et des moments vécus en Pologne dans Les Lubies ou dans La Tapisserie des jours. On voyait souvent en Cazin l'ambassadeur de la culture polonaise. Les critiques polonais y reviennent maintes fois quand ils évoquent ses mérites incontournables. Cette appellation me paraît d'autant plus justifiée quand j'étudie ses œuvres françaises, imprégnées de polonité et incrustées des images de la Pologne. Dans la Préface à $L a$ Tapisserie des jours, Cazin décrit ce livre comme l'histoire « de [s]a vie, de [s]es pensées, de [s]es sentiments au jour le jour dans le courant de toute une année ${ }^{35}$. Dans la trame de cette tapisserie, les fils polonais sont très fréquents. Les noms des écrivains polonais émaillent le texte. On voit Zapolska à côté de Mickiewicz, Józef Wittlin à côté de Hieronim Morstin. Les forêts des Tatras et l'église de Notre-Dame de Cracovie alternent avec le Parc de Pławowice de chez Morstin et les troncs roses des pins à Rogalin. Ces mémoires d'un humaniste en paix se sont nourris des images de Pologne.

Les activités principales de Cazin-traducteur et Cazin-écrivain s'intensifient au cours des années 1930. Je voudrais rappeler une fois encore la grande série de traductions des œuvres polonaises qu'il a publiées - de Weyssenhoff, Berent, Norwid et Mickiewicz à Jarosław Iwaszkiewicz. Il a également travaillé sur Tadeusz Rittner, Z. Krasiński, Słowacki, Zapolska et Boy-Żeleński. En même temps, on attend de lui une multitude de petits travaux pour les amis de l'Ambassade polonaise, tels que des consultations linguistiques, des corrections de textes français produits par les Polonais, des traductions de conférences, comme celle que Beata Obertyńska a donnée à Lisbonne en 1936. À ces occupations diverses, il faudrait ajouter l'élaboration des textes de Jean de Giraudoux que traduisait son ami Jan Lechoń, attaché culturel de l'Ambassade polonaise, deux nouvelles éditions de Pan Tadeusz, pour lesquelles il fallait préparer les notes et l'introduction, enfin des épreuves à corriger qui affluaient sans cesse.

En tant que polonisant attitré, bien que sans chaire universitaire, Cazin devait participer aux nombreuses soirées franco-polonaises et aux réceptions à l'Ambassade de Pologne. Un honneur qui ne manquait pas de le charger d'un effort supplémentaire, dont je voudrais présenter un petit échantillon tiré de son Journal :

Dimanche 14 juin 1936

Levé à $8 \mathrm{~h}$. le cœur dans la bouche pour courir à l'Étoile chez Jean Lechon qui prépare son toast officiel au Jubilé de Rosa Bailly. Ce toast est fort beau. Je sacrifie mon déjeuner au Bois Colombes. Dès $3 \mathrm{~h}$ de l'après-midi je vais me remettre au travail avec ma petite machine. Quelle vie ! ${ }^{36}$

Pourtant son immense effort de traducteur et propagateur de la littérature polonaise ne lui garantissait pas le succès auprès des éditeurs et des directeurs de théâtres. Il a traduit Moralność Pani Dulskiej de Zapolska sous le titre La Moralité de Madame Dulska en 1933 etterminé en octobre 1936 La Comédie non divine de Z. Krasiński ${ }^{37}$ sans avoir trouvé d'éditeur. L'estime de l'Ambassade polonaise ne se traduit pas par des succès matériels. Le 25 octobre, il note dans son Journal, fatigué et déçu: «Travail acharné à la copie de la 
Nieboska », et il ajoute amèrement le jour suivant : «On achève la copie peu avant midi, mais le travail n'est jamais, jamais achevé. Il faut maintenant que je place cette copie. Que je fasse de démarches auprès des directeurs des théâtres... $»^{38}$ Malheureusement, ses démarches n'ont pas abouti. Il a fallu attendre encore plus de soixante à soixante-dix ans pour que les Polonais de France et de Pologne entreprennent ces éditions. Cela peut paraître incroyable, mais dans les mêmes années 1930, Cazin sillonne la France avec de nombreuses conférences sur la littérature polonaise, il participe aux Congrès de slavisants à Cracovie et à Prague, soutient son doctorat à Lviv et voyage en Italie. Il donne aussi une série de conférences à Prague, Brno, Bratislava, Karlsberg. Tout est lié avec la littérature polonaise.

Les glorieuses années 1930 lui apportent le prix du Pen-Club polonais pour la traduction de La Martre et la fille de Weyssenhoff en $1930^{39}$ et pour la traduction de Pan Tadeusz en $1934^{40}$. L'Ambassadeur de Pologne lui offre solennellement La Croix de Commandeur de l'Ordre Polonia Restituta pour la version française de la grande épopée. Cazin devient le héros des cérémonies officielles qui commémorent le centenaire de la publication de Pan Tadeusz à Paris (1834). Dans les fonds Cazin conservés au Musée de la Littérature à Varsovie, il est possible de lire presque toutes les coupures de presse, tous les échos européens de sa traduction qu'il a soigneusement collés dans un épais cahier. $\mathrm{Vu}$ de l'extérieur, c'est une époque de grands triomphes et de grande renommée. Pourtant son Journal révèle des moments de dépression et de grande fatigue.

34 À partir de février 1935, Cazin est chargé de cours de littérature et civilisation au Centre d'Études Polonaises, créé auprès de la Bibliothèque de l'Ile Saint-Louis grâce aux efforts de Franciszek Pułaski. La Pologne Littéraire fournit des informations détaillées sur les fonctions scientifiques du Centre et sur son rôle comparable à l'Institut Français créé à Varsovie en $1925^{41}$. Cazin a eu l'honneur de prononcer la conférence inaugurale intitulée «Le Génie latin et l'esprit français en Pologne ». Fidèle à sa conception de la culture polonaise, l'écrivain s'est brillamment penché sur les liens qui unissent les Polonais avec la civilisation occidentale au cours des siècles ${ }^{42}$. Le Journal de cette époque contient plusieurs réflexions sur des cours qu'il a donnés sur le préromantisme, sur la littérature baroque en Pologne, sur l'ossianisme, etc.

Durant ces années si riches en événements, Cazin revient à sa propre création. Il publie un petit livre charmant, Paul qui roule (1935), où il partage avec les lecteurs ses souvenirs de voyage à travers les différentes régions de la Pologne et de l'Italie, en 1928 et 1932. Notons que ce livre est vraiment digne d'une traduction polonaise. Il nous présente l'image la Deuxième République animée par une vie littéraire et artistique très intense telle que pouvait la voir un Français ayant " deux âmes », toujours sensible aux souvenirs de ses premiers contacts avec le pays et ses habitants. Les perspectives se superposent, le début du siècle se dessine derrière les images des années 1930 et le tout baigne dans l'émotion comme dans ce récit de voyage à Poznań : «C'est dans le beau Rogalin, domaine de Raczyński (...) que je suis né polonisant, voilà plus d'un quart de siècle. J'ai lu pour la première fois les ballades de Mickiewicz sous les arbres centenaires du parc qui ressemblent à des cascades de verdure. ${ }^{43}$

36 Paul qui roule semble s'inscrire aussi bien dans la lignée des journaux intimes que dans la tradition des notes de voyageur. Néanmoins Cazin tend surtout à favoriser les images de la vie intellectuelle qu'il a pu observer et à laquelle il a participé grâce à ses nombreux amis polonais. Il décrit les traditions pascales polonaises et les spectacles à Varsovie, il 
savoure l'atmosphère des cafés littéraires. Il partage avec le lecteur la joie de son émission à la Radio polonaise et de ses aventures durant son voyage à Zakopane. Le texte, incrusté d'observations perspicaces et pleines d'humour, pouvait facilement attirer le public français en lui donnant le goût d'une visite en Pologne.

\section{Entre l'amour de la culture polonaise et la condamnation politique}

Après les changements politiques et idéologiques survenus sur la carte de l'Europe, Cazin s'est trouvé dans une situation difficile. Il ne voulait pas abandonner sa «deuxième patrie ", bien qu'il n'approuvât pas le régime communiste dans celle-ci. Il ne s'est intéressé à la politique ni jadis ni après 1945. C'est pourquoi il n'a pas permis qu'elle s'impose et domine ses pensées après la guerre. Il est resté fidèle à ses amis polonais et aux grands écrivains du passé. Il a renoué aussi de nouvelles amitiés avec Mirosław Żuławski puis avec Tadeusz Breza et sa femme. Cette ouverture a provoqué des accusations douloureuses du coté d'une partie de la presse. Malgré l'atmosphère politique hostile aux échanges avec les pays de l'Est, Cazin s'est donné beaucoup plus aux activités de polonisant que d'écrivain français, se contentant de La Bataille d'Autun (1946) et de petits textes sur la Bourgogne, publiés en 1945 et $1948^{44}$.

De nouvelles charges familiales, l'éducation de ses deux fillettes, ses forces physiques diminuées et le constant manque d'argent rendaient sa vie difficile. Les maisons d'édition n'étaient pas intéressées par la réédition de ses œuvres, considérées comme démodées. Il s'est vu obligé de quitter Autun vers la fin de 1949 pour aller vivre avec sa nouvelle femme en Provence. Il parait évident que cette décision l'a éloigné de son petit monde bourguignon. Il a dû se séparer de sa belle maison, d'une grande partie de ses collections de livres et de beaux objets d'antan. Dans une lettre adressée à Victor Poucelle du 26 février 1951, glissée dans le Journal de 1953, Cazin décrit sa situation d'exilé en Provence :

Ruiné, déchu, déraciné. J'avais à Autun une belle vieille maison, un hôtel Louis XIII, un vrai musée, que Dieu m'avait donné, comme au bonhomme Job et qu'il m'a reprise...

J'ai vécu deux ans sur la vente de mes meubles. Bah... ces biens matériels... Ne m'en irai-je pas bientôt dans un vieux drap ? (...)

Remarié sur le tard - par l'évêque d'Autun, saint et habile jeune homme - deux enfants, deux petites filles : 9 ans et 3 ans... Il fallait qu'elles vivent. À Canossa. À l'Université. Limite d'âge.

Le gouvernement marxiste de Varsovie fonde ici pour moi un lectorat. La Faculté me charge de conférences de littérature comparée. Médiocres traitements. Pas même les Allocations et Assurances du moins tout juste ce temps grâce à une lutte acharnée contre une administration stupide. On tient, on est de ces vieux coriaces bourguignons qui ne se laissent pas tuer sans s'être battus. ${ }^{45}$

Il est évident qu'il devait beaucoup au gouvernement de la Pologne Populaire, qui n'a pas oublié ses mérites de grand traducteur. Comme il l'a mentionné dans la lettre citée, c'était le gouvernement de Varsovie qui lui avait offert le poste de lecteur à l'Université d'AixMarseille (1950-1957). Le gouvernement «marxiste » avait tout intérêt, a très justement précisé Maria Delaperrière, à rendre hommage à ce grand ami de la Pologne, resté en dehors des enjeux politiques ${ }^{46}$. Les activités de polonisant donnaient un sens à la vie intellectuelle de Cazin et lui assuraient des moyens d'existence. 
Le Journal d'Aix-en-Provence des années 1953-1963 prouve à quel point Cazin avait besoin de liens spirituels avec sa patrie culturelle d'adoption pour garder un équilibre et une énergie vitale. À l'âge de soixante-dix ans, il n'acceptait pas de se sentir vaincu. En dépit de relations diplomatiques tendues et pleines de méfiance, Cazin offre toutes ses forces au service de la culture polonaise. Les invitations venues de Pologne lui permettent d'aller à Cracovie en 1948, à Varsovie en 1955 pour le centenaire de la mort de Mickiewicz puis en 1958 pour les débats du comité polonais du prix Nobel (il y propose la candidature de Parandowski.)

Marginalisé, même oublié en tant qu'écrivain en France, il jouit toujours d'une grande considération en Pologne, il est couronné de prix et décoré de médailles, parmi lesquelles il faudrait citer La Croix de Commandeur avec Étoile de l'Ordre de Polonia Restituta, attribuée, comme la presse française l'a annoncé, « par le Conseil d'État à l'occasion de ses cinquante années d'activité en faveur de la connaissance de la littérature polonaise en France et du renforcement de l'amitié franco-polonaise $»^{47}$. Il a été élu Membre Ordinaire de l'Académie Polonaise des Sciences en $1956^{48}$, titre dont il était très fier. Il s'est rendu pour la dernière fois en Pologne en 1960 pour travailler à Nieborów sur la «Présentation » de l'album Pologne $e^{49}$ publié par Hachette.

Abandonné à son sort en France où il vivotait à grand-peine avec sa famille, il a trouvé en Pologne, comme jadis, au début du $\mathrm{xx}^{\mathrm{e}}$ siècle, des gens capables de l'apprécier à sa juste valeur. Son Journal dévoile quelle importance avaient pour lui ces signes de reconnaissance et d'amitié. Il révèle également quelle joie il a ressenti en voyant enfin deux de ses livres traduits en polonais : L'Humaniste à la guerre (en 1957) et L'Homme qui a vu bien de choses (en 1960).

Les écrits intimes de Cazin mettent en évidence qu'il se révoltait contre les attaques d'une partie de la presse prétendant qu'il avait trahi la « cause polonaise ». Dans son Journal, le lundi 13 juillet 1953 à 4 heures et demi du matin, il a noté avec amertume :

Au travail depuis longtemps. Le seul moyen que j'aie de sortir de la misère, c'est de sortir de l'ombre. Je m'accommoderais fort bien de l'ombre dans la sécurité. (...) Cet argent qui me vient de Pologne, n'est pas celui de Staline, comme le croient naïvement les bons lecteurs du Figaro. ${ }^{50}$

44 Il est important d'insister sur le fait que Cazin cherchait désespérément à expliquer sa position de propagateur de la culture polonaise et d'ami de la nation et non des idéologues communistes. Mais il était réaliste, convaincu qu'il fallait respecter les gens qui se débattaient sur ses terres ruinées et qui voulaient y reconstruire la vie. Le Journal de 1953 contient un texte intitulé "Notes sur les affaires polonaises", dans lequel l'auteur entreprend une vive polémique avec ceux qui l'accablaient de jugements sévères. Je voudrais donner la parole à Cazin. Le 9 décembre 1953 il écrit :

...Certes, je préférerais ne pas dépendre de « l'étranger ». Que faire?

Si les Français avaient jugé utiles mes compétences de polonisant, il ne tenait qu'à eux de s'en servir et de m'assurer l'indépendance, en me mettant «au service » d'un organisme français.

C'est le gouvernement polonais qui est venu au secours d'un vieil écrivain français, ruiné, déchu, et qui lui assure son pain.

Il n'a rien demandé de moi, depuis quatre ans, qu'un gouvernement quelconque ne puisse demander d'un professeur de littérature. ${ }^{51}$

Cazin continuait à donner ses cours à l'Université d'Aix, des conférences sur la littérature polonaise à Marseille, Toulon et même Autun, des cours d'été à Nice et ses traductions de Parandowski (Zegar słoneczny qu'il a traduit par Le Cadran solaire en 196152) puis de Breza ( 
La Porte de bronze, $1962^{53}$ et La Démarche ${ }^{54}$, ce dernier livre est sorti après sa mort en 1963). Il publiait de nombreux articles dans la presse, surtout dans La Croix, sur les personnalités célèbres de l'histoire de la Pologne telles que Jan III Sobieski, la reine Hedvige, le prince Józef Poniatowski (1951-1952). Ses études sur la poésie romantique de Słowacki, Mickiewicz et Norwid étaient publiées dans la revue Peuples Amis (1953-1960). Il restait en contact avec les écrivains et les journalistes polonais qui, après les événements d'Octobre 1956, venaient lui rendre visite chez lui à Aix comme Jan Parandowski et sa femme, Janusz OdrowĄż-PieniĄżek, Mirosław Żuławski et Tadeusz Breza. Il n'arrêtait pas d'informer les Français sur les trésors de la littérature polonaise. Et il profitait de chaque occasion pour révéler les liens qui depuis longtemps unissaient les Polonais et les Français.

La découverte d'une vieille tombe au cimetière d'Aix, celle du poète romantique émigré Konstanty Gaszyński, a incité Cazin à faire les démarches pour sa rénovation auprès de l'Ambassade Polonaise. Grâce a son initiative, une cérémonie officielle a été organisée avec la participation de la Mairie d'Aix et des représentants de l'Ambassade. L'événement a attiré l'attention de la presse régionale, ce qui a permis à Cazin de parler de l'amitié séculaire entre les deux nations. Le mercredi 18 novembre 1953 à 5 h du matin il a noté dans son Journal :

... Jour mémorable de dimanche 15 novembre 1953 quand j'entrais dans ce cimetière à la tête de toute une ville : Université, Clergé, Armée, Magistrature à côté des représentants de la Mairie, de la Sous-préfecture, de l'Archevêché - avec les communistes et les Conservateurs aux côté du Ministre représentant de la République Populaire de Pologne - je n'ai pas pensé que c'est moi qui avais amené tout cela, moi, pauvre vieil homme de médiocre situation, qui avais remué tout cela. 55

Cette cérémonie a été relatée dans Le Monde, L'Humanité, La Croix de ParisetLe Courrier d'Aix. Cazin a pu publier plusieurs articles sur Gaszyński. Radio-Marseille a diffusé une interview de lui. Dans le discours qu'il a prononcé au cimetière, il s'appuyait sur «La dimension importante des échanges franco-polonais au cours des siècles ». Il a dit :

Cette pierre tombale, pierre millénaire de l'amitié franco-polonaise, est aussi une pierre de témoignage. Elle témoigne que les peuples peuvent s'aimer puisqu'on a pu inscrire sur le drapeau : "Français et Polonais de tout temps amis ». Ce n'est pas un vain mot. Exemple unique de l'histoire de l'Europe, jamais Français et Polonais n'ont pris les armes les uns contres les autres. (...) Cette tombe nous invite à penser à la Pologne, non seulement avec admiration mais avec reconnaissance. ${ }^{56}$

Ainsi en 1953 Cazin a réussi à rapprocher les deux camps politiques par l'intermédiaire d'un modeste poète, l'ami de Z. Krasiński qui, au milieu du $\mathrm{XIX}^{\mathrm{e}}$ siècle, est devenu le chantre de la Provence.

La participation de Cazin à l'édition prestigieuse de la Maison Hachette du livre intitulé La Pologne. Les Albums du Guide Bleu (1961) constitue en quelque sorte ses adieux avec ce pays et le summum de sa tâche d'ambassadeur de la culture polonaise. Sa " Présentation », il ne faudrait pas le dissimuler, a subi quelques interventions de la part de l'Ambassade polonaise. Mais elle offre au lecteur français un aperçu synthétique sur la Pologne et elle révèle l'histoire intime des relations de l'auteur avec sa deuxième patrie. Le succès du livre s'avère beaucoup plus retentissant à Varsovie qu'à Paris où il s'efface un peu dans la série des guides bleus. Néanmoins, c'est la première présentation de la Pologne complète et bien illustrée. Il n'est pas étonnant que la critique polonaise s'en soit enthousiasmée. Cazin note dans son Journal le 24 juillet 1962 : «Divine chaleur, divine lumière, vents 
apaisés. Joie de recevoir de Varsovie des journaux qui exaltent ma Pologne dont le nom sera à jamais lié à mon œuvre. $»^{57}$

\section{Menand écrit :}

Notre pays n'a pas donné à cet écrivain dont les œuvres sont toutes de finesse et de sensibilité, la place qui lui revient dans notre littérature et dans notre vie nationale. Sa seconde patrie, La Pologne, le place à juste titre parmi nos plus brillants auteurs contemporains et certains de ses contes et essais sont publiés à l'étranger dans l'anthologie des meilleurs écrivains français. ${ }^{61}$

Après la mort de Cazin survenue le 12 juin 1963, les articles de journaux régionaux annonçaient que «les lettres bourguignonnes [étaient] en deuil» en lui témoignant de l'estime et en soulignant comme Paul Chovelon qu'il était « resté profondément attaché à la Bourgogne de ses ancêtres (...)» et qu'il «s'était, toute sa vie appliqué d'une part à célébrer sa province dans divers essais et nouvelles, d'autre part de faire connaître la Pologne par des études, des traductions et des conférences $»^{62}$.

53 Il est incontestable qu'il a toujours été perçu en Pologne comme un "ambassadeur ", « un grand ami », « Paweł Kaziński », « notre cher Monsieur Paul Cazin », « Nasz kochany Paweł » (Notre bien-aimé Paul). Au bord de la Seine comme au bord de la Vistule, on le considérait surtout comme un intermédiaire entre deux littératures et deux civilisations. Après des années de déchirement et de déception, il a accepté ce rôle en y voyant un titre de gloire. Il n'y a rien d'étonnant à ce que la rédaction de Pays de Bourgogne ait publié en couverture sa photo devant l'église des Sacramentines à Varsovie. De toute évidence, ce Français polonisé par la littérature polonaise devait se sentir plus à sa place dans ce vieux quartier de Varsovie que sous les platanes d'Aix ${ }^{63}$. 
54 délégation de Polonais qui est venue en Provence pour lui rendre une visite officielle : le vice président de l'Université de Varsovie Janina Kulczycka-Saloni, l'attaché culturel de l'Ambassade de Pologne, l'écrivain Tadeusz Breza et la lectrice de polonais à l'Université d'Aix Teresa Garnysz-Kozłowska ${ }^{64}$. C'était juste avant l'accident de voiture dont il a été victime le 5 mai 1963. Cette photo semble chargée d'une symbolique qui pourrait me servir de conclusion. À partir de 1904, du début de la maturité intellectuelle de Cazin et jusqu'à ses derniers jours, tant le hasard que ses choix conscients ont fait de lui un Français en exil spirituel en Pologne.

\section{NOTES}

1. L'Association « Les Amis de Paul Cazin » a été créée en décembre 1963 à Autun.

2. Polska była jego wielkA miłościĄ. Listy do Paula Cazina - francuskiego polonofila (La Pologne était son grand amour. Lettres à Paul Cazin, un polonophile français), choix, introduction, commentaire et bibliographie de Chruściński K., Académie Pédagogique de Pomeranie, Słupsk, 2000, 355 p.

3. Les Mémoires de Jean Chrysostome Pasek, gentilhomme polonais (1656-1688), traduit et commenté par Paul Cazin, Société d'édition « les Belles lettres », Paris, 1922, 350 p.

4. Reymont Wł., L'Apostolat du knout en Pologne. Notes de voyage au Pays de Chelm, traduit par Paul Cazin, Perrin, Paris, 1912, 225 p. Voir : Cazin P., «Justice», in : Le Journal des Débats, 3-23 février 1912. Coupure de presses conservée par Cazin, sans mention des pages.

5. Breza T., La Porte de bronze, chronique de la vie vaticane, traduit par Paul Cazin, Julliard, Paris, $1962,480 \mathrm{p}$.

6. Breza T., La Démarche, traduit par Paul Cazin, Julliard, Paris, 1963, 295 p.

7. Mickiewicz A., Pan Tadeusz, traduit par Paul Cazin, Garnier frères, coll. Classiques Garnier, Paris, 1936, $370 \mathrm{p}$.

8. Norwid C. K., Le Stigmate, traduit par Paul Cazin, Gallimard, Paris, 1932, 222 p.

9. Krasiński Z., La Comédie non divine, traduit par Paul Cazin, Noir sur Blanc, Montricher-Paris, 2000, 117 p.

10. La traduction de Fantazy de Juliusz Słowacki, que nous envisageons de faire éditer, est encore sous forme de pages dactylographiées. La Morale de Madame Dulska vient de paraître grâce aux soins de la Faculté de Langue et de Lettres Polonaises de l'Université de Varsovie (Zapolska G., La Morale de Madame Dulska/Moralność pani Dulskiej [traduction de Paul Cazin], KnyszTomaszewska D., BĄbiak G. \& Bocianowski C. (éd.), éd. de l'Université de Varsovie, Varsovie, 2011, 407 p.). Nous avons publié la pièce de Gabriela Zapolska en version bilingue à partir de la seule copie du manuscrit qu'il a fallu déchiffrer, étant donné que le texte dactylographié est à présent introuvable. Cependant, il faudrait préciser que nous avons récemment découvert l'original du manuscrit dans un cahier perdu parmi les livres et les papiers que Mme Catherine Cazin, la fille de l'écrivain, a bien voulu offrir à Faculté de Langue et de Lettres Polonaises de l'Université de Varsovie en 2011.

11. Nous avons publié quelques fragments du journal de Paul Cazin dans les ouvrages qui ont paru dans le courant des années 1990 à l'Université de Varsovie. Voir : Knysz-Tomaszewska D., Suwała H., OdrowĄż-PieniĄżek J., Paul Cazin diariste, épistolier, traducteur, Knysz-Tomaszewska D. (éd.), Institut de Littérature Polonaise de l'Université de Varsovie, Varsovie, 1997, 189 p. ; Id., Dole 
i niedolefrancuskiego polonisty (1881-1963) (La fortune et les infortunes d'un polonisant français), Institut de Littérature Polonaise de l'Université de Varsovie, Varsovie, 1999, 268 p.

12. Cazin P., La Bataille d'Autun, 30 lithographies originales d'André Dulaurens, Saintyves, Autun, 1946. L'ouvrage, publié en tirage restreint, est vite devenu une œuvre rarissime. Il a été réédité par Le caractère en marche éditeur à Génelard en 1995. J'ai pu obtenir un exemplaire grâce à la gentillesse de Jean-Pierre Valabregue. Voir également: Villard M., Ombres et lumières de l'occupation et la libération d'Autun.1940-1944, Autun 1984, 368 p. Voir encore le texte basé sur la documentation de Cazin : Suwała H. "L'Occupation allemande vue à travers les textes de Paul Cazin ", in : Paul Cazin diariste, épistolier, traducteur, Op. cit., pp. 29-41 ; Knysz-Tomaszewska D., "Paul Cazin témoin intime du temps historique», in : Le témoignage dans la littérature polonaise du XX ${ }^{e}$ siècle, Konicka H. \& Zaremba Ch. (éd.), Institut d'études slaves, Paris, 2008, pp. 119-127.

13. Weyssenhoff J., La Martre et la fille, traduit par Paul Cazin, Gallimard, Paris, 1930, 315 p.

14. Boy-Żeleński T., «Paul Cazin czy Paweł Kaziński » (Paul Cazin ou Paweł Kaziński), in : Kurier Poranny, Varsovie, 9 novembre 1934. Réédité in: Boy-Żeleński T., Pisma. Felietony (Æuvres. Feuilletons), Henryk Markiewicz (éd.), t. III, Państwowy Instytut Wydawniczy, Varsovie, 1959, p. 129.

15. Il est nécessaire de rappeler que le jeune élève de Cazin est devenu un diplomate renommé puis Président de la Pologne en exil de 1979 à 1986. Cette lettre est conservée dans la collection privée du destinataire.

16. Raczyński E., Rogalin i jego mieszkańcy (Rogalin et ses habitants), The Polish Research Centre, Londres, 1964, $227 \mathrm{p}$.

17. Cette lettre a été écrite le 14 mars dans le domaine de Laszki, près de Bobrówka, où Cazin séjournait chez les Zamoyski. Voir : Archives «parisiennes» de Cazin, Musée de la Littérature Adam Mickiewicz à Varsovie, dossier $n^{\circ} 125$.

18. Cazin P., "La parole est aux traducteurs. Une enquête de la Pologne littéraire », in : Pologne littéraire, 15 octobre-15 novembre 1933, p. 2.

19. Cazin P., «Europejskie echa Pana Tadeusza» (Les échos européens de Pan Tadeusz), in: Ilustrowany Kurier Codzienny, n 271, 30 septembre 1934, p. 2.

20. Cazin P., «Le Roman polonais au XIX siècle », in : La Revue du Mois, t. XIX, no 109, 10 janvier 1915, pp. 73-98.

21. Zapolska G., L'Oraison dominicale, traduit par Paul Cazin, Bibliothèque internationale d'édition, Paris, 1908, 231 p.

22. Weyssenhoff J., Vie et opinions de Sigismond Podfilipski, traduit par Paul Cazin, Plon-Nourrit et Cie, Paris, 1911, 229 p.

23. Voir note 4 .

24. Reymont Wł., Justice, traduit par Cazin P. et Jacquet A., Picart, Paris, 1925, 215 p.

25. Żeromski E., « L'Ombre », in : La Vie, nº 28-41, 1912.

26. Ziejka F., "Paul Cazin à Paris, capitale artistique polonaise », in : Actes du Colloque Paul Cazin 1881-1963, Nawrocki A. \& Claudon Fr.(dir.), Colloque «Paul Cazin 1881-1963 », 21-22 novembre 1997, Dijon-Autun, éd. Conseil régional de Bourgogne, Dijon, 1998, pp. 104-105.

27. Ibid., p. 105.

28. Maritain J., Théonas ou les entretiens d'un sage et de deux philosophes sur diverses matières inégalement actuelles, Bibliothèque française de philosophie, Nouvelle Librairie Nationale, Paris, 1925 , p. 49.

29. Ibid., p. 50.

30. Cru J. N., Témoins. Essai d'analyse et de critique des souvenirs de combattant édités en français de 1915 à 1928, Les Étincelles, Paris, 1929, p. 108.

31. Archives « parisiennes », dossier $n^{\circ} 113$.

32. Idem.

33. Journal d'Autun, le 19 octobre 1930, in : Archives « parisiennes », dossier $n^{\circ} 126$. 
34. La version française de son doctorat a été publiée en 1940 : Le Prince-évêque de Varmie Ignace Krasicki 1735- 1801, Bibliothèque Polonaise, Centre d'Études Polonaises de Paris, Paris, 1940, 319 p.La traduction polonaise date de 1983 : KsiĄż ̨̇ biskup warmiński Ignacy Krasicki 1735-1801 [trad. par Mroziński M.], Goliński Z. (éd.), Olsztyn, 1983 [deuxième éd. 1986], 465 p.

35. Cazin P., La Tapisserie des jours, Librairie Plon, Paris, 1934, pp. 1-2.

36. Fragments du Journal de 1934 à 1936 concernant la Pologne, copiés par Juliette Cazin, Musée de la Littérature Adam Mickiewicz à Varsovie.

37. Voir note 10.

38. Ibid.

39. Voir note 14.

40. Voir note 8 .

41. Klingsland Z. St., "L'inauguration du centre d'Études Polonaises à Paris ", in: Pologne littéraire, $\mathrm{n}^{\circ} 103,15$ avril 1935, p. 2

42. Cazin P., Le Génie latin et l'esprit français en Pologne, Conférence donnée à l'inauguration du Centre d'Études Polonaises de Paris, Bibliothèque Polonaise de Paris, Société française de Librairie, Gebethner et Wolff, Paris 1935, 22 p.

43. Paul Cazin, Paul qui roule, (Pologne-Italie), illustrations de Jaques Vallery Radot, éd. J. Gigord, Paris, 1935, pp. 34-35.

44. Cazin P., Forestier H. \& Tisserand R., Veillées de Bourgogne, lithographies de Jacques Grange, Michel Jeanniard éditeur, Autun, 1945, 215 p. ; Cazin P., Paysages et types de Bourgogne, quarante lithographies originales du peintre André Dulaurens, Autun, 1948, $166 \mathrm{p}$.

45. Journal de 1953, in : Archives « parisiennes », dossier $n^{\circ} 127$.

46. Delaperrière M., «Autour de Paul Cazin. Les relations culturelles entre la France et la Pologne au Xx ${ }^{\mathrm{e}}$ siecle », in : Colloque international Paul Cazin 1881-1963, Op. cit.,p. 87.

47. Le Monde, Paris, 2 décembre 1955.

48. Lettre officielle du 25 juin 1956 signée par Henryk Jabloński et Wacław Sierpiński.

49. Cazin P., "Présentation ", in: Pologne. Les Albums des guides Bleus, Hachette, Paris, 1961, pp. 5-31.

50. Archives " parisiennes », dossier $n^{\circ} 127$.

51. Notes sur les affaires polonaises jointes au journal de 1953, in : Idem.

52. Parandowski J., Le Cadran solaire, traduit par Cazin P., Deligne S. \& Marcq M., Del Duca, coll. UNESCO d'auteurs contemporains, Paris, 1961, 240 p.

53. Voir note 6.

54. Voir note 7.

55. Idem.

56. Cazin P., "À la mémoire de Constantin Gaszynski patriote polonais et journaliste aixois », discours prononcé devant sa tombe au Cimetière d'Aix-en-Provence le 15 Novembre 1953, in: Courrier d'Aix, 21 novembre 1953; "Une Tombe polonaise en France", in: Le Monde, Paris,13 novembre 1953; "Grób poety. List z Provansji " (La tombe d'un poète. Lettre de Provence), in : Przegląd Kulturalny, n 50, 1953 ; «Un Poeta polacco giornalista in Provenza » (Un poète polonais journaliste en Provence), in : Ricerche Slavistiche, ${ }^{\circ} 3$, Rome, 1954. Coupures de presses conservées par Cazin, sans mention des pages.

57. Archives « parisiennes ", dossier $n^{\circ} 136$.

58. OdrowĄż-PieniĄżek J. "Les Archives "varsoviennes" de Paul Cazin et quelques souvenirs personnels ", in : Paul Cazin diariste, épistolier, traducteur,Op. cit., p. 105.

59. Cité d'après : Pays de bourgogne, nº 37, 1962, p. 485.

60. "Allocution de M. Paul Cazin. Président du Congrès ", publiée dans : Mémoires de l'Académie des Science, Arts et Belles Lettres de Dijon, t. CXV, Dijon, 1965,p. 17. Le texte de cette allocution est resté inachevé. La mort a empêché Cazin d'en rédiger la version définitive.

61. Menand J., «L'Ami autinois », in : Pays de bourgogne, no 31, 1962, p. 482. 
62. Chovelon P., « Paul Cazin est mort », in : Le provençal, 13 juin 1963.

63. Voir : Na ścieżkach pamięci. Album Paula Cazina (Sur les sentiers de mémoire. L'Album de Paul Cazin), Choix de documents iconographiques et de textes par Knysz-Tomaszewska D., BĄbiak G. P. (éd.), Faculté de Langue et de Lettres Polonaises, Université de Varsovie, Varsovie, 2009, 200 p.

64. Cette photo accompagne l'article de Paul Chovelon, Op. cit.

\section{INDEX}

Index géographique : Aix-en-Provence, Autun, France, Paray-le-Monial, Pologne

Index chronologique : communisme, Deuxième Guerre mondiale, entre-deux-guerres, XXe siècle oeuvretraite Breza T. : Spiżowa brama, Breza T. : Urząd, Cazin P. : Décadi ou la pieuse enfance, Cazin P. : L'Alouette de Pâques, Cazin P. : L'Homme qui a vu bien de choses, Cazin P. : L'Humaniste à la guerre, Cazin P. : La Bataille d'Autun, Cazin P. : La Tapisserie des jours, Cazin P. : Les Lubies, Cazin P. : Paul qui roule, Krasiński Z. : Nie-Boska komedia, Mickiewicz A. : Pan Tadeusz, Norwid C. K. : Stygmat, Parandowski J. : Zegar słoneczny, Pasek J. Chrz. : Pamį̨tniki, Reymont Wł. : Sprawiedliwie, Reymont Wł. : Z ziemi chełmskiej, Weyssenhoff J. : Soból i Panna, Weyssenhoff J. : Żywot i myśli Zygmunta Podfilipskiego, Zapolska G. : Modlitwa Pańska, Zapolska G. : Moralność Pani Dulskiej, Żeromski St. : Cień

Mots-clés : littérature française, littérature polonaise, traduction littéraire

\section{AUTEUR}

DANUTA KNYSZ-TOMASZEWSKA

Université de Varsovie 